Marylise Cottet, Anne Rivière-Honegger et Hervé Piegay

\title{
Mieux comprendre la perception des paysages de bras morts en vue d'une restauration écologique : quels sont les liens entre les qualités esthétique et écologique perçues par les acteurs?
}

\begin{abstract}
Avertissement
Le contenu de ce site relève de la législation française sur la propriété intellectuelle et est la propriété exclusive de l'éditeur.

Les œuvres figurant sur ce site peuvent être consultées et reproduites sur un support papier ou numérique sous réserve qu'elles soient strictement réservées à un usage soit personnel, soit scientifique ou pédagogique excluant toute exploitation commerciale. La reproduction devra obligatoirement mentionner l'éditeur, le nom de la revue, l'auteur et la référence du document.

Toute autre reproduction est interdite sauf accord préalable de l'éditeur, en dehors des cas prévus par la législation en vigueur en France.
\end{abstract}

\section{revues.org}

Revues.org est un portail de revues en sciences humaines et sociales développé par le CLEO, Centre pour l'édition électronique ouverte (CNRS, EHESS, UP, UAPV).

\section{Référence électronique}

Marylise Cottet, Anne Rivière-Honegger et Hervé Piegay, « Mieux comprendre la perception des paysages de bras morts en vue d'une restauration écologique : quels sont les liens entre les qualités esthétique et écologique perçues par les acteurs? ", Norois [En ligne], 216 | 2010/3, mis en ligne le 01 décembre 2012. URL : http:// norois. revues. org/index3408.html

$\mathrm{DOI}$ : en cours d'attribution

Éditeur : Presses universitaires de Rennes

http://norois.revues.org

http://www.revues.org

Document accessible en ligne à l'adresse suivante : http://norois.revues.org/index3408.html Ce document est le fac-similé de l'édition papier.

Cet article a été téléchargé sur le portail Cairn (http://www.cairn.info).

\section{CAIRN}

Chercher. Repérer. Avancer.

Distribution électronique Cairn pour Presses universitaires de Rennes et pour Revues.org (Centre pour l'édition électronique ouverte)

(c) Tous droits réservés 


\title{
MiEUX COMPRENDRE LA PERCEPTION DES PAYSAGES DE BRAS MORTS EN VUE D'UNE RESTAURATION ÉCOLOGIQUE : QUELS SONT LES LIENS ENTRE LES QUALITÉS ESTHÉTIQUE ET ÉCOLOGIQUE PERÇUES PAR LES ACTEURS?
}

\author{
Marylise Cottet, Anne Rivière-Honegger, Hervé Piegay \\ UMR 5600 «Environnement, Ville, Société », CNRS \\ (Université de Lyon 3), \\ 18 rue Chevreul - 69007 Lyon \\ marylise.cottet@univ-lyon3.fr,honegger@club-internet.fr,herve.piegay@ens-lyon.fr
}

\begin{abstract}
RÉSUMÉ
Le contexte actuel des restaurations écologiques demande de porter une attention particulière à la perception du public. Il est en effet désormais bien admis que la sensibilisation du public à la restauration des écosystèmes et, en définitive, la pérennité des actions engagées, dépendent en partie de l'apparence du milieu après travaux. La qualité du paysage constitue donc un indicateur de succès des projets de restauration. Plus largement, le paysage constitue un vecteur de diffusion des enjeux environnementaux auprès du public.

La mise en ceuvre de restaurations de bras morts sur le flewve Rhône et sur la rivière d'Ain a motivé la réalisation d'une enquête de perception. Elle porte précisément sur un objet bien spécifique: les plans d'eau de ces bras morts. Deux objectifs prévalent dans le cadre de cette étude: (1) Mettre en évidence les facteurs explicatifs de la perception des plans d'eau de bras morts; (2) Comprendre si les connaissances environnementales conditionnent, ou non, les préférences. Ces interrogations sont nécessaires dans la perspective d'une définition concertée des futurs projets de restauration.

Une enquête par photo-questionnaires a donc été menée. Les résultats confirment que certaines variables relatives à l'aspect de l'eau expliquent en grande partie les préférences. En effet, certaines induisent des jugements positifs (transparence de l'eau, végétation aquatique dont la forme est bien définie, contrastes de couleurs, etc.) tandis que d'autres engendrent davantage des jugements négatifs (opacité, sédiments, végétation aquatique dont la forme est mal définie, etc.). Des analyses multivariées ont permis de définir une typologie de plans d'eau en fonction de leur perception (4 types visuels). Ces résultats concrets permettent d'envisager une modélisation de la perception des plans d'eau de bras morts. Elle consisterait à anticiper les réactions esthétiques à partir d'un jeu de variables concernant l'apparence de l'eau. Cette perspective semble prometteuse dans le cadre d'une gestion participative des zones humides.
\end{abstract}

MotS CLÉ : Perception - paysage - eau - esthétique - santé - qualité - bras morts restauration - acteurs - Rhôn - Ain 


\section{ABSTRACT \\ Better understanding perception of floodplain lakes landscapes for an eco- logical restoration: what are the links between aesthetical and ecological qua- lities?}

The current context of ecological restorations requires specific attention to public perception. The public's adhesion to any given restoration project is instrumental in the success and the durability of its operations. This adhesion depends partly on the appearance of the environments after the engineering works have been performed. Landscape quality is thus an indicator of success for restoration projects. Widely, landscape may be considered as an efficient tool to communicate the environmental stakes to the public.

Within the context of the floodplain lakes restorations in the Upper Rhône and Lower Ain Rivers, a perception survey has been conducted which focussed on water appearance. Two main objectives were defined: (1) defining the structural criteria which explain the floodplain lakes perceptions; (2) defining if environmental knowledge influences - or not - landscape preferences. These are necessary considerations in order to establish a dialogue between stakeholders when elaborating the restoration goals.

The survey used photo-questionnaires. The results confirm that some criteria concerning the water appearance explain a large part of preferences. Some criteria (transparency of water, well shape-defined aquatic vegetation, colour-contrasted lakes, etc.) induce positive judgments whereas some other criteria (opacity of water, bad shape-defined aquatic vegetation, presence of sediments, etc.) imply negative assessments. Thanks to multivariate analysis, a typology of floodplain lakes was defined as a function of their perception (4 visual types). These concrete results open new perspectives. A model of the floodplain lakes perception could be built in order to anticipate the aesthetic reactions as a function of a set of water appearance criteria. It would be an efficient tool in order to initiate a participative management of wetlands

KEY WORDS : Perception - landscape - water-aesthetics - health-quality-floodplain lakes - restoration - stakeholders - Rhône River - Ain River

La rivière d'Ain et le Rhône français se caractérisent par la présence de nombreux bras morts. Issus de la dynamique fluviale, ces milieux créent les conditions favorables au développement de nombreuses communautés aquatiques et terrestres, favorisant ainsi le maintien de la biodiversité. Leur disparition, au cours du siècle dernier, a conduit à la mise en œuvre d'ambitieux projets de restauration. Compte tenu des modifications paysagères que ces opérations impliquent et des premiers retours d'expérience, il apparaît opportun de mieux comprendre la perception qu'en a le public. Il est en effet désormais bien admis que la sensibilisation du public à la restauration écologique des milieux et, en définitive, leur pérennité, dépend en partie du rendu visuel de ce milieu après travaux. La qualité du paysage constitue donc un indicateur de succès des projets de restauration. Plus largement, le paysage apparaît comme un vecteur de diffusion des enjeux environnementaux auprès du public.

Pourtant les connaissances relatives à la perception paysagère des écosystèmes sont encore limitées. Comprendre cette perception du public est un enjeu majeur afin de parvenir à une gestion durable des écosystèmes qui soit partagée par les acteurs. Tel est le but de cette recherche, appliquée à un paysage particulier, celui des bras morts d'un grand système fluvial européen : le fleuve Rhône et son affluent, la rivière d'Ain. Ces deux cours d'eau ont fait l'objet, entre 1995 et 2005, d'ambitieux projets de restauration écologique (projet de restauration hydraulique et écologique du Rhône en 1995, programme LIFE Nature sur l'Ain en 2002 et programme décennal de restauration du Haut-Rhône en 2003). D’autres projets de ce type sont envisagés sur la 
partie aval du Rhône et sur d'autres cours d'eau comme le Rhin, le Doubs, la Loire ou encore le Danube. Le développement de connaissances relatives à la perception des paysages de bras morts constitue donc un apport précieux dans la perspective opérationnelle de ces prochains projets de restauration écologique ${ }^{1}$.

Ainsi, on tentera de comprendre et d'expliquer les jugements esthétiques portés sur ces paysages aquatiques et à les relier à des causes explicatives. Plus précisément, deux objectifs prévalent :

Mettre en évidence les préférences esthétiques du public en termes de plans d'eau de bras morts et en comprendre les facteurs explicatifs, qu'ils soient écologiques ou visuels.

Comprendre si ces perceptions sont universelles ou si, à l'inverse, avoir des connaissances écologiques conditionne les préférences des écosystèmes aquatiques. De fait, la perception des experts est-elle compatible avec celle du public?

\section{Problématisation : Pourquoi s'intéresser à la perception paysagère dans le contexte des projets de restauration écologique?}

\section{UNE NÉCESSAIRE PRISE EN COMPTE DES DIMENSIONS SOCIALES ET CULTURELLES POUR LA DÉFINITION DES PROJETS DE RESTAURATION ÉCOLOGIQUE}

Afin de pallier la dégradation des écosystèmes, des politiques de restauration ont vu le jour au cours des deux dernières décennies. Il est désormais bien admis que la gestion des écosystèmes doit reposer sur un projet de société, prenant en compte les différentes aspirations sociales (Blandin et Bergandi, 2000; Mouratiadou et Moran, 2007 ; Nassauer et al., 2001). L'adhésion du public constitue en effet un facteur déterminant du succès d'un plan de gestion (Giordano et al., 2005). Cette nécessaire construction concertée des projets est d'autant plus vraie dans le cas des politiques de restauration des écosystèmes. Ces dernières demandent l'instauration de références et le choix d'indicateurs. Si l'on s'en tient à la définition donnée par E. Le Floch et J. Aronson, l'écosystème de référence représente " une approximation de l'état souhaitable, comme une norme choisie parmi plusieurs états alternatifs possibles » (Le Floc'h et Aronson, 1995). Ce dernier est donc une construction sociale, reposant sur des valeurs, des représentations du «bon état écologique ». Dans ces conditions, il semble pertinent de s'appuyer en partie sur des indicateurs transcrivant les composantes culturelles et sociales des écosystèmes (Charles et Kalaora, 2003; Donadieu, 2002). Or jusqu’à présent, les indicateurs retenus sont essentiellement d'ordre naturel, qu'ils soient écologiques, géomorphologiques, chimiques, ou biologiques (Amoros et al., 2000; Bazin et Barnaud, 2002; Trémolières et al., 2002). Il apparaît dès lors essentiel d'élargir la conception de la restauration et d'y intégrer les dimensions historique, culturelle, sociale, politique, morale, esthétique (Higgs, 1997), ouvrant la voie à une restauration «éco-sociétale » (Cairns, 1995) ou «éco-culturelle ( Higgs, 1997). Mais, l'implication des sociétés dans la définition des projets de restauration n'est pas chose aisée, d'autant qu'aucune prescription ni aucune orientation ne sont données par la législation (Mouratiadou et Moran, 2007). Il existe indiscutablement des difficultés pour concilier les objectifs définis par des experts environnementaux sur la base de connaissances rationnelles, et les aspirations sociales basées, non sur une connaissance objective, mais sur une image subjective du réel (Ibery et al., 1982; Junker et Buchecker, 2008). Dès lors, il convient de mettre en œuvre de nouvelles stratégies pour connaître et comprendre les représentations et les comportements vis-à-vis des écosystèmes pour parvenir à un consensus (De Vanssay, 2003).

1. Ce travail de recherche a été réalisé dans le cadre d'une thèse, intitulée «Étude de la perception des paysages de bras morts dans un contexte de restauration écologique. Cas du fleuve Rhône et de la rivière d'Ain ", co-dirigée par A. Honegger et H. Piégay. Il s’inscrit par ailleurs dans le programme de recherche Ingeco (INEE, CNRS) « restauration des zones humides fluviales : de la prévision à la mesure du bénéfice écologique et social », coordonné par G. Bornette, 2007. 
Marylise Cottet, Anne Rivière-Honnegger, Hervé Piegay

\section{LE PAYSAGE, UN OUTIL DE SYNTHÈSE ENTRE LES IMPÉRATIFS ÉCOLOGIQUES ET LES ASPIRATIONS SOCIALES : VERS UNE « ESTHÉTIQUE ÉCOLOGIQUE »?}

Le paysage peut être considéré comme un moyen d'associer les sociétés à la gestion des écosystèmes. Défini par la Convention Européenne du paysage en 2000 comme « une partie de territoire telle que perçue par les populations, dont le caractère résulte de l'action de facteurs naturels et/ ou humains et de leurs interrelations », le paysage constitue le produit de l'expérience perceptive. Dans la mesure où les hommes appréhendent les phénomènes à l'échelle de leur expérience directe (Gobster et al., 2007), le paysage apparaît comme une échelle pertinente pour analyser les liens qui existent entre les sociétés et les écosystèmes (Boulton, 1999; Cairns, 1995; Gobster et al., 2007). La prise en compte de ce lien entre perception sociale des paysages et caractéristiques écologiques n'est pas neuve. En particulier, elle a été analysée sous l'angle des relations entre esthétique et écologie du paysage. En effet, selon de nombreux auteurs, l'expérience esthétique (issue de la perception) induit une motivation sociale à agir en faveur des écosystèmes : plus un environnement est jugé beau, plus il est estimé légitime d'être protégé. On peut donc penser que le paysage apparaît comme un vecteur de diffusion des enjeux environnementaux auprès du public (Cairns, 1995; De Vanssay, 2003; Gobster et al., 2007; Junker et Buchecker, 2008; Nassauer, 1992 ; Nassauer et al., 2001). Dès 1988 avec J. Koh (Koh, 1988), relayé ensuite par de nombreux auteurs (Daniel, 2001 ; Gobster et al., 2007; Nassauer, 1992; Nassauer et al., 2001 ; Parsons, 1995 ; Spirn, 1988; Thorne et Huang, 1991) est créée la notion « d'esthétique écologique ». Selon cette dernière, il convient d' «aligner les objectifs écologiques et les expériences esthétiques pour parvenir à des paysages durables sur les plans culturels et écologiques » (traduit de Gobster et al., 2007). En d'autres termes, l'esthétique écologique suppose de dépasser les appréciations esthétiques basées sur le pittoresque, parfois incompatibles avec la qualité écologique, et de refonder une appréciation esthétique de la nature conforme à un idéal écologique. Dans le contexte des politiques de restauration, cette notion d'esthétique écologique prend tout son sens : compte tenu des modifications paysagères que ces opérations impliquent, une attention particulière doit être portée par les acteurs à la perception du public. Les indicateurs retenus doivent être non seulement écologiques mais aussi esthétiques afin de gagner l'adhésion sociale aux projets et de favoriser la pérennité des actions engagées.

\section{DES CONNAISSANCES EN MATIÈRE DE PERCEPTION SOCIALE DES ÉCOSYSTÈMES À APPROFONDIR}

Mais pour cela, il convient de développer les connaissances en matière de perception esthétique des écosystèmes par le public : vaste chantier qui s'est ouvert au cours des années 1990. Si le champ des études de perception paysagère est ancien, les premières études datant des années 1960 (Linton, 1968 ; Lowenthal et Prince, 1965 ; Shafer, 1969), celui qui s’intéresse plus spécifiquement au lien entre esthétique et écologie est beaucoup plus récent. Les résultats déjà publiés à ce sujet concernent des milieux naturels spécifiques tels que les forêts (Barro et Bright, 1998), les cours d'eau et leurs paysages riverains (Junker et Buchecker, 2008; Nassauer et al., 2001) (Cossin, 2008), les zones humides (Nassauer, 2004), les parcs urbains (Gobster et Barro, 2000; Raffetto, 1993), etc. D'autres résultats se rapportent à des objets environnementaux particuliers tels que le bois mort en rivière (Le Lay, 2007; Le Lay et Piégay, 2007; Mutz et al., 2006), les sédiments dans les cours d'eau (Le Lay et al., à paraître). C'est dans cette continuité que le présent article s'inscrit. Il s'attache à mettre en évidence la perception sociale d'un paysage particulier, celui des zones humides fluviales (ou bras morts). Au sein de ce paysage, un objet précis retiendra plus spécifiquement l'attention : l'eau. Quoi de plus familier que cet élément paysager? Il est désormais bien connu que l'eau influence positivement la perception paysagère (Arriaza et al., 2004; Dearden, 1985; Herzog, 1985; Herzog et Bosley, 1992; Real et al., 2000 ; Ulrich, 1981 ; Yamashita, 2002 ; Yang et Brown, 1992). Pourtant, si l'on se place dans la perspective d'une meilleure compréhension des liens entre esthétique et écologie des milieux aquatiques, de nouvelles recherches sont 
nécessaires. En effet, les connaissances mettant en lien perception de l'eau et environnements aquatiques sont limitées. Seules quelques études consacrées plus spécifiquement à la perception de la qualité de l'eau ont couplé ces évaluations esthétique et écologique (Brody et al., 2005; Ibery et al., 1982; Moser, 1984 ; Smith et Davies-Colley, 1992; Steinwender et al., 2008). Les résultats ont confirmé que les personnes enquêtées utilisent avant tout des critères visuels pour estimer la qualité de l'eau. Ce constat confirme que l'apparence joue un rôle majeur dans l'appréciation de la qualité des milieux aquatiques. C'est à partir de ce constat qu'a été construite l'enquête de perception présentée ici.

\title{
Protocole de l'enquête : l'utilisation de photo-questionnaires
}

\author{
UN CHOIX D'ENQUÊTE SPÉCIFIQUE POUR L'ÉTUDE \\ DES PERCEPTIONS PAYSAGÈRES : LE PHOTO-QUESTIONNAIRE
}

Une enquête par photo-questionnaire a été menée sur internet auprès de plus de 400 personnes de Juin à septembre 2008. Cette technique d'enquête, largement répandue dans le champ thématique de la perception paysagère, consiste à présenter une série de photographies à partir desquelles les personnes interrogées doivent émettre un jugement, généralement d’ordre esthétique. Utilisés principalement depuis les années 1980, les photo-questionnaires ont fait l'objet de nombreuses recherches méthodologiques dont la plupart ont validé l'efficacité de la méthode : les jugements émis à partir de photographies sont comparables aux jugements émis au sein même du paysage, en contexte réel (Shuttleworth, 1980; Vining et Orland, 1989; Zube et al., 1987).

\section{ModAlités D'ÉCHANTILlonNAge DES PERSONNES ENQuÊTÉES ET DES PHOTOGRAPHIES UTILISÉES}

Les personnes retenues dans le cadre de cette enquête appartiennent à des groupes prédéfinis : étudiants en géographie, en écologie ou issus de disciplines n'ayant aucun lien avec les thématiques environnementales; gestionnaires de l'environnement; et enfin, chercheurs ou experts dans le domaine environnemental (fig. 1).

Ces personnes ont été classées plus spécifiquement en fonction de leur connaissance ou de leur absence de connaissance en écologie des milieux humides (données collectées par le biais d'une question incluse au questionnaire : "Avez-vous des connaissances en phyto-écologie des milieux humides?»). Ces échantillons ont été retenus afin d'évaluer la variabilité des opinions entre culture vernaculaire et culture savante et de mettre en évidence ce qui fait (ou non) consensus. Selon la bibliographie, un groupe d'une trentaine de personnes suffirait à obtenir des résultats fiables en termes de perception paysagère (Daniel et Boster, 1976; Stamps, 1992). A. E. Stamps (1992) a en effet réalisé des simulations bootstrap afin de comparer les corrélations inter-groupes d'échantillons de différentes tailles. Un groupe de 25 à 30 personnes suffirait selon lui à obtenir des corrélations de 0,90 minimum. Les effectifs des réponses recueillies dans le cadre du photoquestionnaire satisfont largement ces critères de validité.

Les 16 photographies de l'enquête se rapportent à des vues en gros plan de plans d'eau de bras morts $^{2}$. Ces vues ont été choisies de manière à représenter au mieux les différents écosystèmes présents au sein des bras morts de l'Ain et du Rhône (photo 1 - planche XI). Pour cela, les photographies ont été échantillonnées selon deux variables, écologique et visuelle. La première se rapporte au niveau trophique de l'écosystème. Cette variable est en effet la base des classifications de bras morts élaborées par certains écologues et permet, combinée à d'autres variables écologiques, de déterminer la durée de vie d'un écosystème de bras mort (Amoros et al., 2000). Le critère visuel, quant à lui, repose sur 4 types de plans d'eau définis d'un point de vue formel : réfléchissant,

2. Cette enquête, centrée sur les plans d'eau de bras morts, est complémentaire à une autre enquête, visant à étudier la perception de grands paysages de bras morts : cette dernière repose sur un photo-questionnaire, présentant des vues larges de paysages. Pour plus de détails, se reporter au travail de thèse mentionné plus haut. 


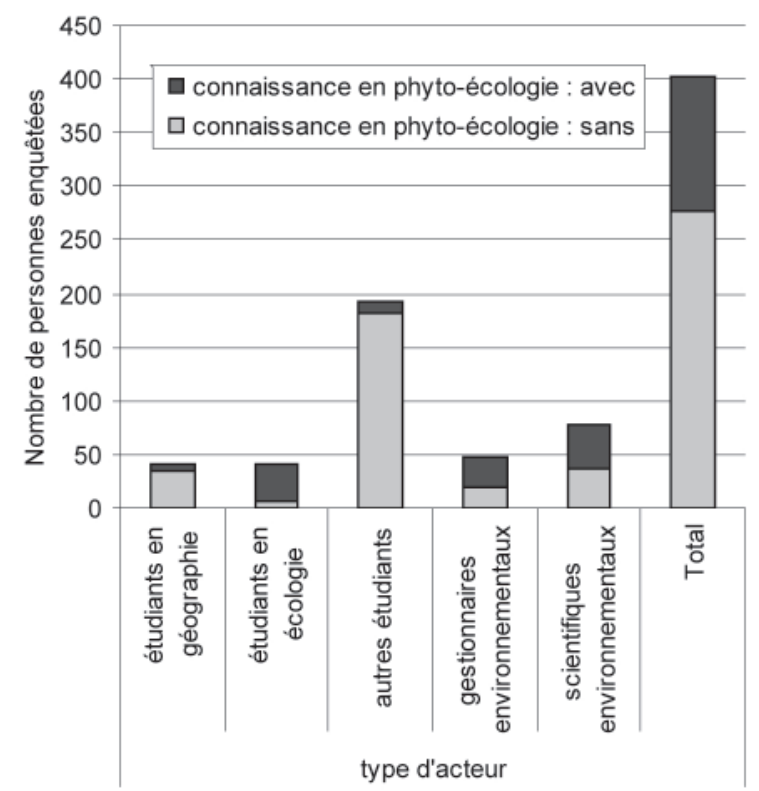

transparent avec sédiments (grossiers et fins), avec végétation aquatique flottant à la surface, et avec végétation aquatique ancrée au fond du plan d'eau. Les effectifs de chacun de ces types visuels sont définis dans le tableau 1.

Figure 1 : Échantillons de populations soumises à l'enquête

Surveyed population

\begin{tabular}{|l|c|l|c|}
\hline \multicolumn{2}{|c|}{ Critère visuel } & \multicolumn{2}{c|}{ Critère écologique (trophie) } \\
\hline \multicolumn{1}{|c|}{ Type } & Effectif & \multicolumn{1}{c|}{ Type } & Effectif \\
\hline Réfléchissant $(\mathrm{R})$ & 3 & Eutrophe $(\mathrm{E})$ & 4 \\
\hline Transparent avec sediments $(\mathrm{T})$ & 3 & Mesotrophe $(\mathrm{M})$ & 5 \\
\hline Végétation aquatique de surface $(\mathrm{Vs})$ & 4 & Oligotrophe $(\mathrm{O})$ & 3 \\
\hline Végétation aquatique de profondeur $(\mathrm{Vp})$ & 6 & Type trophique inconnu $(\mathrm{I})$ & 4 \\
\hline
\end{tabular}

Tableau 1 : Critères écologique et visuel utilisés lors de la sélection des photographies

Ecological and visual criteria used for the sampling of photographs

\section{UN RECUEIL ET UN TRAITEMENT DE DONNÉES QUANTITATIVES : VERS UNE HIÉRARCHISATION DES PERCEPTIONS SOCIALES}

La mise en œuvre de traitements quantitatifs dans le cadre de cette étude répond à des intentions bien précises : (1) être en mesure de hiérarchiser les préférences esthétiques relatives aux plans d'eau de bras morts; (2) identifier les facteurs explicatifs de cette perception et leurs influences respectives; (3) comparer les perceptions propres à différents groupes sociaux.

Ainsi, dans un premier temps, il a été demandé aux participants d'évaluer ces 16 photographies en fonction de deux variables subjectives : la beauté et la santé des plans d'eau. L'évaluation des photographies s'appuie sur une échelle visuelle analogique qui permet le recueil de données quantitatives continues (Le Lay et al., 2005; Lukasiewicz et al., 2001 ; Piégay et al., 2005). Cette acquisition élargit les possibilités en termes de traitements statistiques permettant en particulier de mieux appréhender la variation inter-groupe en s'appuyant sur des notes moyennes pour répondre aux questions posées dans le cadre de cette étude. Dans un second temps, les personnes enquêtées avaient pour consigne de caractériser ces 16 plans d'eau par des termes de leur choix. 
Les différents termes mentionnés ont été traités par analyse thématique de contenu (logiciel Modalisa). D’après B. V. Berelson (1954) «L'analyse de contenu est un examen objectif, exhaustif, méthodique et, si possible, quantitatif d’un matériel constitué par des communications verbales [... en vue d'en classer et d'en interpréter les éléments. » Une simplification et une interprétation des représentations propres aux écosystèmes aquatiques, tels sont les objectifs de cette étude. Ainsi, les 5000 termes cités ont été classés en 57 catégories et un traitement quantitatif a pu être envisagé à partir des occurrences de chacune de ces catégories. Au terme de l'analyse, des traitements statistiques exploratoires ont été effectués (ACP et CAH en particulier). Les liens entre caractéristiques objectives des plans d'eau et jugements subjectifs (sensations et sentiments éprouvés) ont alors pu être établis et une typologie a pu être construite : quatre types de plans d'eau de bras morts ont été mis en évidence.

\section{Résultats}

\section{LES MECANISMES REGISSANT LA PERCEPTION DES BRAS MORTS}

\section{Un lien indiscutable entre santé perçue et esthétique du plan d'eau}

L'objectif de la gestion des écosystèmes est avant tout de garantir un état écologique satisfaisant. Ce «bon état écologique » est souvent traduit auprès du public en termes de «santé » des milieux. Même si ce concept est parfois critiqué pour la simplification excessive qu'il réalise à propos du fonctionnement des écosystèmes (Callicott, 1995; Jamieson, 1995; Simberloff, 1998 ; Suter, 1993), il est utile dans la mesure où il est directement interprété par le public (Boulton, 1999). Ainsi, dans la perspective d'une gestion concertée, il apparaît important de comprendre ce qui fait, aux yeux du public, un écosystème en bonne santé.

Cette question a été posée dans le cadre de l'enquête. Pour les milieux aquatiques de bras morts, le résultat apparaît indiscutable : un plan d'eau en bonne santé est un beau plan d'eau (fig. 2). On observe en effet une très bonne corrélation entre les évaluations données pour la beauté et la santé $\left(\mathrm{r}^{2}=0,91\right)$.

Mais au-delà du lien existant entre beauté et santé, comment sont caractérisés ces milieux?

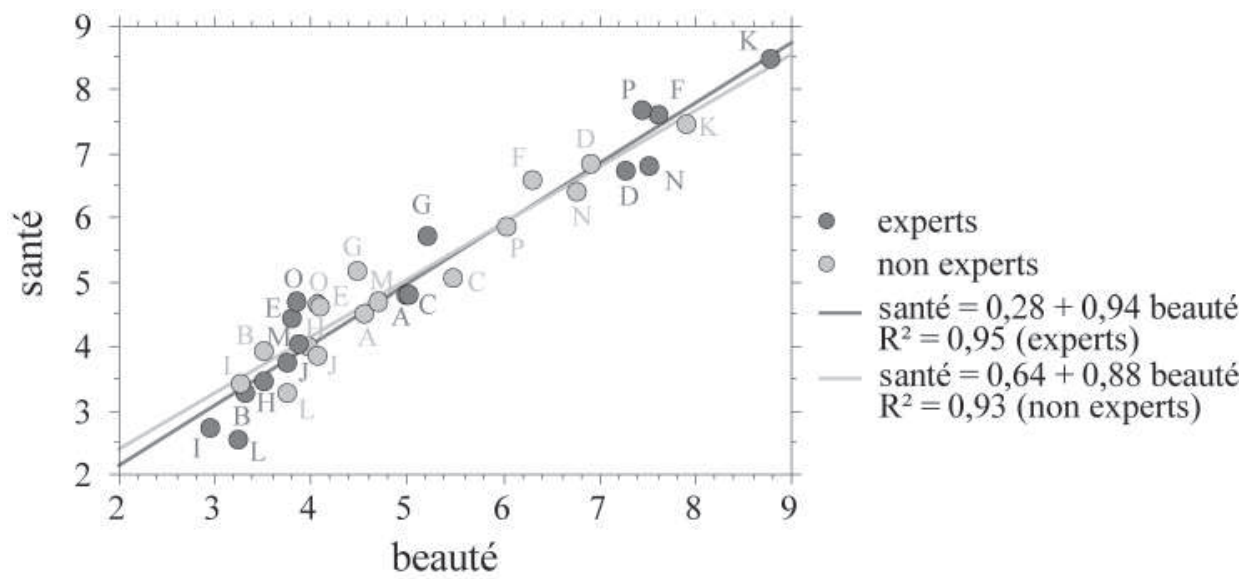

Figure 2 : Régression linéaire entre les variables de beauté et de santé perçues Linear regression between beauty and judged health criteria 
Marylise Cottet, Anne Rivière-Honnegger, Hervé Piegay

\section{Des préférences marquées}

Si l'on considère les notes attribuées à chacune des photographies, il existe d'importantes variations (fig. 2). Ainsi, la population enquêtée a des préférences marquées pour les plans d'eau K, F, P, N, D. Au contraire, les plans d'eau I, L, B, H, et dans une moindre mesure, J M, E, O, sont jugés plutôt inesthétiques. Quant aux autres, ils occupent une situation intermédiaire. Si l'on se réfère à la planche de photographies présentée en photo 1 , on peut constater que les groupes mis en évidence ont certaines caractéristiques semblables. On peut donc supposer qu'il existe des variables visuelles qui expliquent ces différences d'appréciation. Il convient donc de valider cette hypothèse et de comprendre quelles sont les variables impliquées dans la perception sociale des plans d'eau de bras morts.

L'analyse des termes cités pour caractériser chacune des photographies est alors susceptible d’apporter des éléments de réponse.

\section{QUELS LIENS ENTRE LES ATTRIBUTS PHYSIQUES DES PLANS D'EAU ET LES PREFEFENCES ESTHETIQUES?}

\section{La caractérisation des écosystèmes aquatiques}

Le langage constitue un outil de communication permettant l'expression des représentations sociales. D’après B. De Vanssay (2003), « nommer, c'est déjà représenter ». Il a ainsi été demandé aux personnes participant à l'enquête d'associer à différents plans d'eau des mots de leur choix : afin de ne pas lasser les répondants, cet exercice ne leur a été proposé que pour les deux plans d'eau les mieux appréciés et les deux les moins estimés (ces préférences étant définies à partir des notes d'esthétique).

Le choix d'utiliser une question ouverte dans le questionnaire («Citez trois mots qui caractérisent ce plan d'eau ») répondait à la volonté de ne pas orienter les réponses et de laisser une certaine liberté aux participants pour caractériser le plan d'eau. La même logique a prévalu lors de la définition des thèmes utilisés dans l'analyse : les thèmes n'ont pas été définis a priori mais l'ont été au fur et à mesure de la lecture des mots mentionnés. Ainsi, les thèmes retenus se font l'écho de la perception des participants et ne correspondent en rien à des clés de lecture créées a priori.

Cinq grandes catégories se distinguent pour caractériser un plan d'eau, chacune d'entre elles correspondant à des niveaux de subjectivité différents :

1. Les objets : on observe en premier lieu des termes qui se rapportent à des objets présents sur les photographies (algues, pollens, sédiments, etc.). Ce premier niveau ne fait appel à aucune subjectivité. Il retranscrit directement ce que l'observateur identifie.

2. Les processus environnementaux : cette catégorie se rapporte à l'identification de processus écologiques ou géomorphologiques, résultant d’une expertise réalisée à partir d'éléments visibles sur les clichés (eutrophisation, sédimentation, pavage, etc.)

3. Les sensations : ont été classés dans cette catégorie tous les thèmes liés à des expériences sensorielles directes, qu'il s'agisse d'une expérience visuelle, olfactive ou tactile (clarté/opacité; luminosité; homogénéité/contraste; température; odeur, etc.). Cette catégorie intègre un premier degré de subjectivité : elle fait intervenir la perception et peut demander une certaine interprétation de la part des répondants.

4. Les sentiments : cette classe recouvre tous les termes transcrivant une émotion liée à la perception du plan d'eau (beauté, mystère, tristesse, surprise, etc.). Les termes classés au sein de cette catégorie témoignent de processus psychologiques, totalement subjectifs.

5. Les activités : cette dernière catégorie englobe des termes évoquant des activités suggérées par la perception du plan d'eau (pêche, baignade, etc.). Elle traduit une abstraction importante dans la mesure où elle suppose une projection de l'individu dans le milieu. 


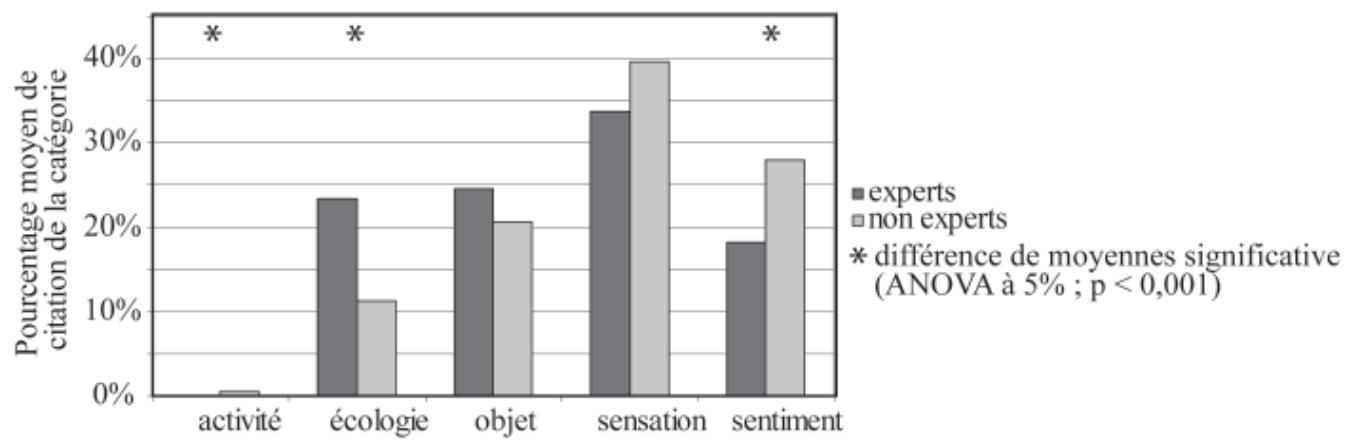

Figure 3 : Part des catégories utilisées pour caractériser les plans d'eau de bras morts Proportion of categories used to characterize the floodplain lakes waters

La fréquence de ces différentes catégories a été calculée (fig. 3). Il apparaît que les « sensations » sont les plus citées pour caractériser un plan d'eau, suivies par les " sentiments », les « objets », les " processus environnementaux », et enfin, loin derrière, les « activités ». Les représentations (sensations, sentiments) sont prépondérantes sur les attributs " réels » (objets et processus environnementaux), témoignant ainsi de la force évocatrice des plans d'eau. Enfin, les plans d'eau de bras morts ne suggèrent que très rarement la pratique d'activités. Ce constat est susceptible d'avoir des répercutions importantes en termes de gestion. En effet, la préservation des écosystèmes est envisagée depuis une, voire deux décennies, en fonction des services qu'ils rendent aux sociétés (Millennium Ecosystem Assessment, 2005). Ainsi, au regard du public, les environnements de bras morts ne favorisent que faiblement les activités de loisirs. Doit-on en conclure que ces milieux apportent, selon les personnes interrogées, des services d'autre nature (protection contre les crues, maintien de la biodiversité, etc.) ? Ou bien ce constat est-il le signe que les bras morts sont devenus, de par leur délaissement, des espaces ignorés dont la richesse locale est méconnue?

Une analyse plus fine des contenus a été conduite afin de savoir si des groupes de photographies peuvent être identifiés en fonction des items cités. Y a-t-il notamment un lien entre les éléments perceptibles d'une part et les sentiments et les sensations exprimées d'autre part?

Typologie issue des perceptions sociales : 4 types de bras morts fluviaux

Afin de ne pas simplifier à outrance l'analyse des perceptions et d'en comprendre toute la complexité, les multiples items ont été regroupés en 57 thèmes. La fréquence de chacun d'entre eux a été calculée photographie par photographie et la structure du tableau correspondant ( 57 thèmes x 16 photographies) a fait l'objet d'une analyse multivariée exploratoire. Une analyse factorielle des correspondances (AFC) a été menée afin de comprendre les correspondances qui existent entre les différentes photographies. Une classification ascendante hiérarchique (CAH) a ensuite été réalisée dans le but de mieux comprendre le lien entre ces groupes de photographies et la fréquence des thèmes.

Les résultats de l'AFC (fig. 4) permettent d'expliquer plus de la moitié de l'information sur les deux premiers axes (les valeurs propres sont de 30,12 \% sur l'axe 1, de 21,31\% sur l'axe 2). On observe des correspondances importantes entre certaines photographies (fig. 4). Ainsi, sur les axes 1 et 2, quatre groupes de plans d'eau semblent s'individualiser. Le premier regroupe les plans d'eau $\mathrm{E}, \mathrm{O}$ et $\mathrm{M}$; le deuxième les plans d'eau $\mathrm{B}, \mathrm{I}$, L et $\mathrm{A}$; le troisième les plans d'eau $\mathrm{F}, \mathrm{K}, \mathrm{D}$, $\mathrm{P}$, et $\mathrm{N}$; et enfin, le dernier, les plans d'eau J, H, C, et G.

Les individus (thèmes) se répartissent bien sur les axes. Ainsi, à chaque groupe de plans d'eau correspondent des qualificatifs spécifiques. La mise en relation des caractéristiques physiques des plans d'eau et des termes évoqués par le public semble donc envisageable, favorisant ainsi une 


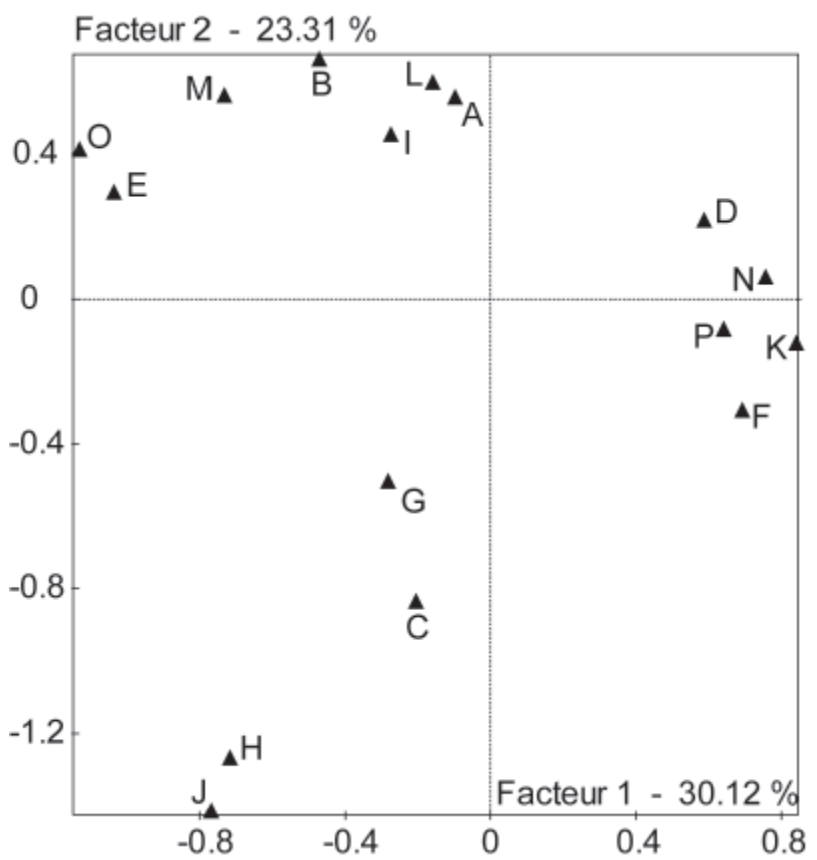

Figure 4 : Plan factoriel F1 x F2 issue de l'AFC

F1 $x$ F2 factorial map resulting from the FCA

lecture socio-écologique des zones aquatiques des bras morts. Cependant, étant donnée la densité du nuage de points, il convient de simplifier l'information. Pour cela, une classification ascendante hiérarchique $(\mathrm{CAH})$ a été mise en œuvre. Le dendrogramme issu de l'analyse est représenté en figure 5. Dans la mesure où quatre groupes de plans d'eau ont été identifiés grâce à l'AFC, quatre classes sont retenues pour découper le dendrogramme. Le détail des classes (termes constitutifs et plans d'eau appariés) est synthétisé dans la figure 6 (planche XII). Les moyennes des notes d'esthétique correspondant à chaque classe y figurent également. D’après le test de Kruskal-Wallis, elles sont significativement différentes $(\mathrm{p}<0,0001)$. On peut donc penser qu'à chaque type issu de la caractérisation visuelle est associé un jugement esthétique spécifique.

La classe la moins appréciée sur le plan esthétique est la classe 2 (moyenne de beauté = 3,7/10). Le point commun entre ces plans d'eau apparaît nettement : tous sont perçus comme des écosystèmes dégradés ( "écologie dégradé »). Ils apparaissent en effet aux yeux des personnes interrogées comme pollués ( écologie pollution oui »), eutrophisés ( écologie trophie forte »), et mal oxygénés (« écologie oxygène »). Cela se traduit sur le plan de l'expérience par des sensations désagréables, qu'elles soient tactiles ou olfactives ( sensation toucher désagréable »; " sensation odeur désagréable»). En définitive, en plus de jugements inesthétiques («sentiment beauté non »), la perception de tels écosystèmes évoque le dégoût ( «sentiment dégoût»), la saleté ( sentiment propreté non »), et fait naître un sentiment d'inquiétude, de danger («sentiment danger/inquiétude »).

Avec une moyenne d'esthétique de 4,1/10, la classe 1 est également peu appréciée. Elle regroupe des vues de plans d'eau où l'eau, jugée profonde ( objet profondeur ») couvre entièrement la photographie (« objet eau »; « sensation vue taille»). Par conséquent, les personnes interrogées s'attardent davantage sur les couleurs du plan d'eau ("sensation vue couleur marron »; " sensation vue clarté non ») ou sur des petits détails tels que des dépôts de surface (objet dépôts surface / débris) ou des vaguelettes («sensation vue dynamique oui »). Il émane donc une grande homogénéité ("sensation vue homogénéité oui ») qui est souvent interprétée en terme de monotonie, voire de tristesse ( «sentiment monotonie/tristesse»). Cependant, ce sont avant tout les plans d'eau de cette classe qui évoquent la pratique de certaines activités, telles que la pêche ou la baignade 


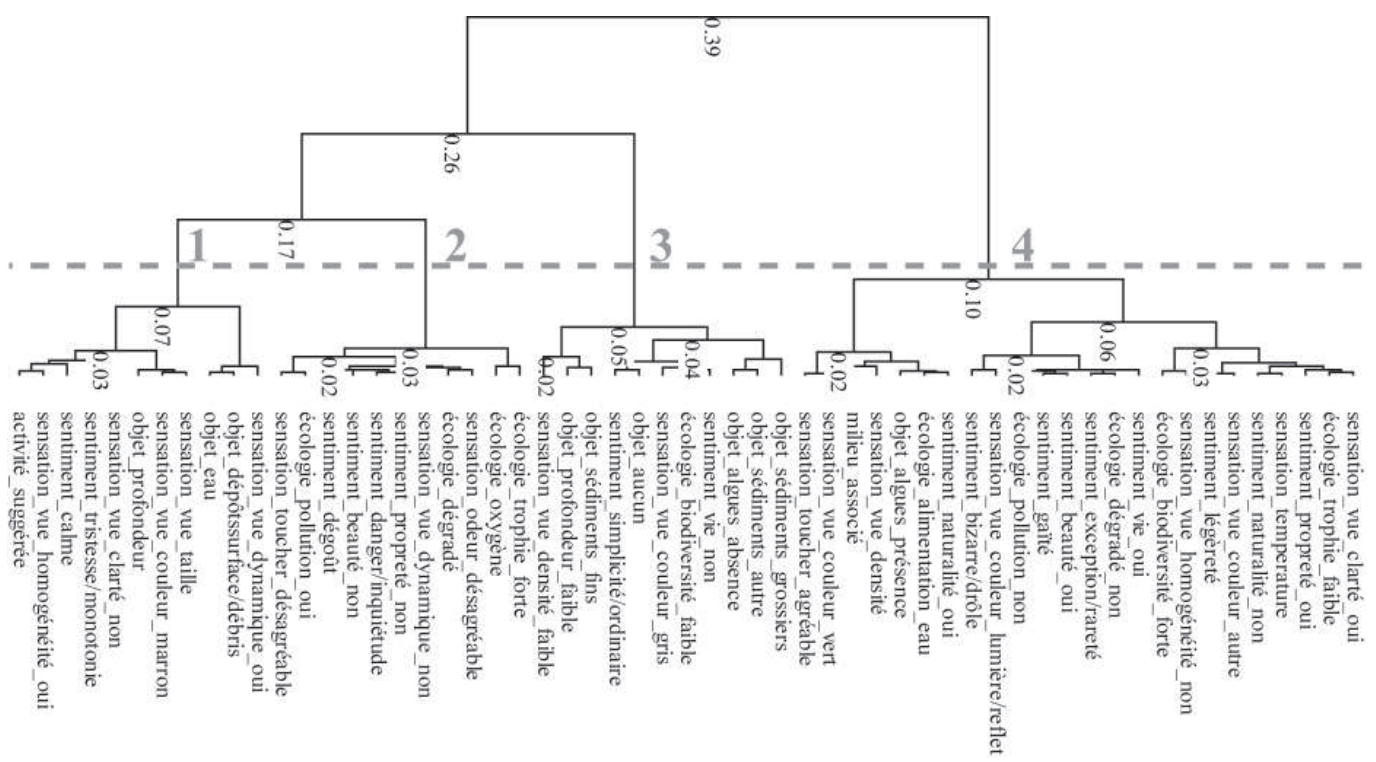

Figure 5 : Dendrogramme issu de la CAH

Dendrogramm resulting from the $\mathrm{HAC}$

(« activité suggérée ») peut-être du fait de leur profondeur, ou encore du sentiment de calme qu’ils diffusent («sentiment calme»).

Les plans d'eau appartenant à la classe 3 ont une note esthétique qui est proche de la moyenne $(4,5 / 10)$. Le point commun de ces plans d'eau est évident : la présence de sédiments, fins ou grossiers («objet sédiments fins »; " objet sédiments grossiers »; « objet sédiments autre »), largement visible du fait de la faible profondeur de l'eau (« objet profondeur faible »). Il émane de cette présence une tonalité très grise des photographies (« sensation vue couleur gris »). Le caractère minéral du plan d'eau (« objet algues absence »; « sensation vue densité faible ») semble globalement plutôt mal perçu par les personnes interrogées. En effet, elles y associent une biodiversité faible (écologie biodiversité faible) et une absence de vie au sein du milieu («sentiment vie non »). A tel point que pour certains, c'est comme s'il n'y avait « rien à voir» («objet aucun»). Au final, c'est un sentiment de banalité, de simplicité, d'ordinaire ( «sentiment simplicité/ordinaire ») qui domine.

Enfin, largement en tête des préférences des participants, les plans d'eau de la classe 4 se singularisent avec une moyenne d'esthétique de 7,1/10. Les objets qui semblent retenir toute l'attention des personnes interrogées sont les végétaux aquatiques (" objet algues présence », "sensation vue couleur vert»), qui baignent dans une eau claire et limpide ("sensation vue clarté oui »). Cela évoque des milieux très « jardinés » (« sentiment naturalité non »), tels que des herbiers d'aquarium. La végétation aquatique apporte, du point de vue des perceptions, une couleur (« sensation vue couleur autre »), une lumière ( « sensation vue couleur lumière »), un contraste ( "sensation vue homogénéité non »), une épaisseur («sensation vue densité forte ») qui n’avaient pas été exprimés jusqu'alors. Ainsi, la perception de tels plans d'eau est très stimulante sur le plan visuel, mais aussi tactile («sensation toucher agréable »). Ces expériences agréables sur le plan sensoriel ont un écho sur le plan émotionnel : les personnes interrogées perçoivent bien sûr de la beauté (« sentiment beauté oui »), mais aussi de la gaîté ("sentiment gaieté »), de la légèreté ("sentiment légèreté »), de la surprise suite à la découverte de nouveautés (« sentiment bizarre/drôle »). Ces sentiments semblent être suscités par l'harmonie perçue des tons et des structures. Par ailleurs, les plans d'eau intégrés à cette classe sont jugés en bon état sur le plan écologique ("écologie dégradé non »). En effet, ils sont perçus comme non pollués («écologie pollution non »), et comme fonctionnels, 
grâce à un renouvellement régulier des conditions physico-chimiques (" écologie alimentation eau »; « écologie trophie faible »). De fait, les répondants perçoivent ces écosystèmes comme étant propres («sentiment propreté oui »), ces derniers incarnant à leurs yeux des milieux éminemment naturels («sentiment naturalité oui »), propres à accueillir des espèces aussi variées qu'exceptionnelles ( «écologie biodiversité forte »; « sentiment vie oui »; « sentiment exception/rareté »).

Ainsi, à partir d'un échantillon de photographies relativement représentatives des écosystèmes aquatiques de bras morts de l'Ain et du Rhône, on a pu identifier quatre types de plan d'eau bien définis du point de vue des perceptions individuelles. Cependant, ces types sont-ils perçus de la même manière par tous? En particulier, les personnes ayant des connaissances en écologie des milieux humides réagissent-elles à la vue de ces plans d'eau comme les personnes n'ayant pas ces connaissances spécifiques?

\section{COMPARAISON DES PERCEPTIONS ENTRE LES EXPERTS ET LES NON EXPERTS}

Lorsque l'on analyse les termes cités pour caractériser les plans d'eau, on peut constater des différences manifestes entre les deux groupes. En particulier, si l'on considère la figure 3, les «non experts » semblent utiliser avant tout des termes issus de la perception et de la cognition ( "sensations » et « sentiments») pour caractériser les milieux (68\%), alors que les « experts» semblent beaucoup plus enclins à employer des termes caractérisant des phénomènes observables (48\% pour les catégories « objet » et « processus environnementaux»). Ce constat est particulièrement sensible en ce qui concerne la catégorie « processus environnementaux » qui est deux fois plus représentée chez les « experts » que chez les «non experts » (fig. 3). De fait, une analyse factorielle discriminante a été réalisée afin de discriminer les termes cités préférentiellement par les experts et les non experts. Cette analyse s'est avérée hautement significative : l'ensemble des individus est en effet correctement classé par la fonction discriminante (taux de resubstitution sur les données d'apprentissage de $0 \%$ ). Les résultats confirment l'utilisation différentielle des termes entre les deux groupes. Le tableau 2 présente les coefficients standardisés des fonctions discriminantes canoniques. On observe, d'après celui-ci, que les experts utilisent des termes beaucoup plus variés que les non experts pour qualifier les écosystèmes aquatiques. Les termes liés à l'expérience visuelle (la clarté de l'eau notamment) et à l'expérience tactile, sont essentiellement cités par les non experts. Au contraire, les termes décrivant une dégradation écologique (dégradation, pollution) ou une richesse écologique (biodiversité) sont mentionnés avant tout par les experts. Suite à cette analyse, il est possible d'identifier des correspondances entre les caractérisations expertes et non expertes (les premières apparaissent en noir dans le tableau 2 et les secondes, en gris). À titre d'exemple, lorsque les non experts décrivent une eau claire, les experts associent à cette eau une faible trophie et la jugent non dégradée. Cette observation est révélatrice d'une différence de perception induite par la connaissance environnementale. Les personnes ayant des connaissances spécifiques en écologie interprètent les photographies de plans d'eau à la manière d'un diagnostic : elles caractérisent les écosystèmes à partir d'objets ou de processus environnementaux identifiables sur les photographies. Au contraire, les personnes n'ayant pas ces connaissances se fient davantage à leur perception sensorielle.

Malgré cette différence fondamentale, les préférences esthétiques semblent très consensuelles : que l'on ait ou non des connaissances environnementales, les mêmes évaluations esthétiques sont portées sur les écosystèmes aquatiques de bras morts. Le test de Mann Whitney réalisé sur la variable des notes d'esthétique en fonction des deux groupes d'acteurs (avec et sans connaissance en écologie des milieux humides) s'est en effet révélé non significatif ( $p>0,05)$. Par ailleurs, la régression observée entre l'esthétique et la santé des milieux perçue chez les personnes sans connaissance spécifique est aussi significative, sinon meilleure, que celle établie par les personnes ayant des connaissances environnementales (fig. 2). Les mêmes modalités de perception semblent donc s'exprimer en matière d'esthétique des écosystèmes aquatiques, que l'on ait ou non des connaissances en matière d'écologie des milieux humides. 
Tableau 2 : Coefficients standardisés des fonctions discriminantes canoniques issus de l'AFD (en maigre, les termes davantage cités par les experts; en gras, termes davantage cités par les non experts)

Standardized coefficients of the canonical discriminant functions resulting from the DFA (in light, words more cited by the experts; in bold, words more cited by the non-experts)

\begin{tabular}{|c|c|}
\hline Variable & F1 \\
\hline écologie_dégradé & $-19,468$ \\
\hline objet_aucun & $-19,153$ \\
\hline écologie_pollution_oui & $-18,607$ \\
\hline milieu_associé & $-13,840$ \\
\hline objet_profondeur & $-13,456$ \\
\hline écologie_dégradé_non & $-11,821$ \\
\hline écologie_biodiversité_forte & $-11,442$ \\
\hline objet_algues_présence & $-10,719$ \\
\hline sensation_vue_couleur_gris & $-7,672$ \\
\hline écologie_oxygène & $-6,438$ \\
\hline écologie_trophie_faible & $-6,077$ \\
\hline objet_profondeur_faible & $-6,006$ \\
\hline activité_suggérée & $-5,373$ \\
\hline écologie_biodiversité_faible & $-4,301$ \\
\hline objet_eau & $-3,748$ \\
\hline sensation_vue_couleur_autre & $-3,342$ \\
\hline objet_sédiments_grossiers & $-2,850$ \\
\hline écologie_pollution_non & $-1,811$ \\
\hline sensation_temperature & 0,075 \\
\hline objet_sédiments_fins & 0,336 \\
\hline écologie_alimentation_eau & 0,671 \\
\hline sensation_odeur_désagréable & 0,835 \\
\hline objet_sédiments_autre & 1,871 \\
\hline objet_dépôtssurface/débris & 2,988 \\
\hline sensation_toucher_agréable & 3,196 \\
\hline objet_algues_absence & 3,863 \\
\hline écologie_trophie_forte & 4,534 \\
\hline sensation_vue_clarté_oui & 9,208 \\
\hline sensation_vue_clarté_non & 10,524 \\
\hline sensation_toucher_désagréable & 12,108 \\
\hline
\end{tabular}

\section{Discussion}

UNE PERCEPTION DES ÉCOSYSTÈMES SUSCEPTIBLE DE FAVORISER DES ACTIONS CONDUISANT À UN APPAUVRISSEMENT DE LA DIVERSITÉ RÉGIONALE

Les environnements de bras morts ont pu être divisés en quatre classes d'après la perception qu'en ont les individus interrogés. Ces classes issues d'évaluations subjectives trouvent-elles un écho sur le plan de l'état écologique objectif des milieux?

Plusieurs travaux d'écologues se sont intéressés à la classification des milieux de zones humides. L'une d'entre elles retiendra notre attention, celle de C. Amoros et al. (2000). En effet, les auteurs 
ont mis au point une méthodologie visant à évaluer la qualité écologique des bras morts (et plus spécifiquement leur durée de vie) à partir de la végétation aquatique qui se développe au sein du milieu. D’après eux, deux facteurs majeurs déterminent la durée de vie d'un système de bras mort : le degré trophique et la perturbation du milieu par les crues. Ces deux facteurs exerçant une forte influence sur le développement des espèces de plante, il est possible de déduire la durée de vie potentielle d'un milieu donné à partir des espèces présentes dans ce milieu. À la lueur des résultats de cette étude, la caractérisation écologique des plans d'eau utilisés dans l'enquête a été mise en œuvre. Elle est synthétisée dans le tableau 3.

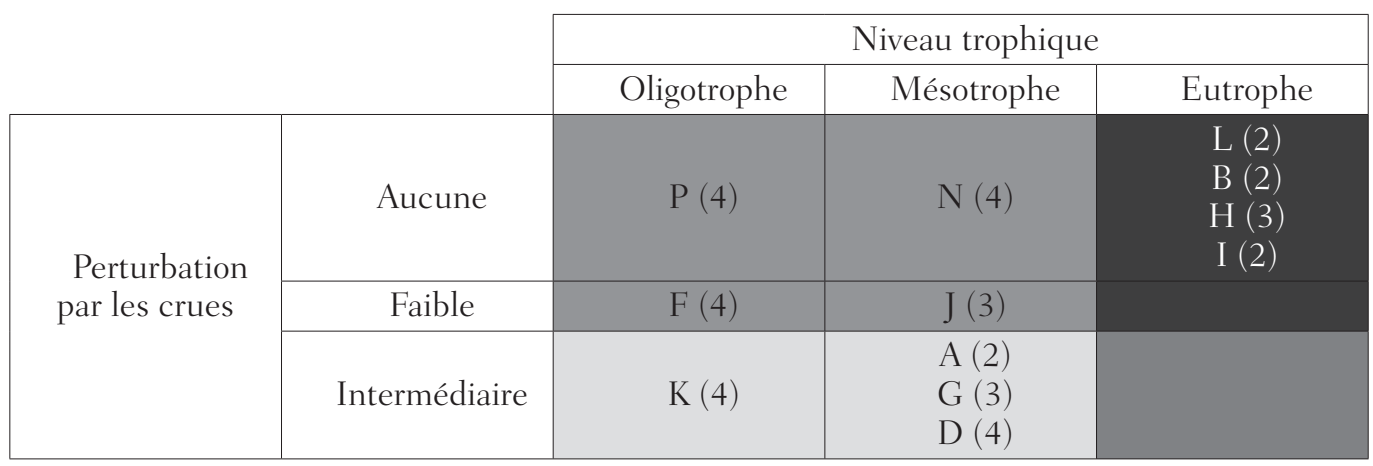

Tableau 3 : Caractérisation écologique des bras morts utilisés dans l'enquête, selon la classification de Amoros et al. (2000); la trame retranscrit la durée de vie des milieux (gris clair = longue; gris moyen = moyenne; gris sombre = courte); les numéros entre parenthèses indiquent la classe de perception à laquelle appartient le plan d'eau

Objective ecological characterization of floodplain lakes used in the survey, from the classification of Amoros et al. (2000); the frame indicates the life span of the environments (light grey = long; mean grey=mean; dark grey=short); the numbers indicate the class of perception of the floodplain lake

On peut observer une certaine correspondance entre la classification fondée sur des indicateurs écologiques (traduits en termes de durée de vie) et celle plus subjective reposant sur des classes de perception. En effet, les milieux dont la durée de vie est très faible ont été classés majoritairement dans la classe de perception 2 (gris sombre). Celle-ci regroupe les plans d'eau jugés dégradés ou pollués. A l'inverse, les plans d'eau appartenant à la classe de perception 4, plans d'eau jugés en très bon état écologique, correspondent systématiquement à des écosystèmes dont la durée de vie est estimée à moyen ou à long terme (gris clair et moyen). Quant aux plans d'eau correspondant à la classe de perception 3, auxquels on associe une biodiversité faible, leur classification écologique est moins homogène : ils se répartissent dans les classes allant d'une durée de vie faible à longue.

Ainsi, la correspondance entre les perceptions et l'état écologique déduit d'une expertise, est réelle. Les bras morts ayant une longue durée de vie sont jugés beaux et en bonne santé tandis que les bras morts dont la durée de vie est faible sont jugés peu esthétiques et en mauvaise santé. Ces résultats concordent avec la littérature scientifique (House et Sangster, 1991; Junker et Buchecker, 2008; Steinitz, 1990). Ainsi B. Junker et M. Buchecker (2008) se veulent rassurant quant aux actions entreprises par les gestionnaires de l'environnement. La corrélation positive qui est observée entre la qualité écologique et les préférences esthétiques indique, selon eux, qu'il n'y a aucune crainte à avoir quant à l'accueil du projet par le public : la moindre action de restauration a des répercutions positives sur le plan esthétique et sera donc, à terme, appréciée

Malgré tout, il convient de rester prudent quant aux conclusions à apporter dans le cadre de la présente étude : le lien entre perception esthétique et qualité écologique mis en évidence n'est pas forcément positif dans la mesure où il n'agit pas toujours en faveur de la préservation des écosystèmes. S'il est vrai que les écosystèmes eutrophisés et de faible durée de vie peuvent correspondre à des milieux dégradés ou pollués, ce n'est pas toujours le cas. Or, ils sont systématiquement interprétés comme tels. Le vieillissement d'un écosystème de bras mort (et donc la diminution 
de sa durée de vie) est pourtant un processus naturel. Il n'est donc pas anormal de rencontrer des écosystèmes vieillissants, généralement eutrophisés. La perception selon laquelle les écosystèmes en fin de vie correspondent à des milieux pollués ou dégradés est problématique dans un contexte de gestion car elle peut conduire à privilégier certains types d'écosystèmes (oligotrophes et mésotrophes notamment) aux dépens d'autres. Les milieux eutrophes, évolués, sont ainsi peu valorisés. Or, ils contribuent à la diversité écologique qu'il est nécessaire d'envisager à une échelle régionale. Ainsi, il est nécessaire de définir avec prudence les objectifs de gestion des écosystèmes. Afin de maximiser la biodiversité régionale, il peut être nécessaire d'élaborer des projets de gestion allant à l'encontre des perceptions communes. Les opérations de restauration qui sont projetées sur la section aval du fleuve Rhône et qui visent à restaurer des écosystèmes eutrophes constituent un bon exemple de la dissociation entre les objectifs de gestion et les perceptions. De tels projets nécessitent que des informations plus détaillées soient transmises au public afin d'expliquer les démarches engagées.

\section{UNE CONVERGENCE DES PRÉFÉRENCES ENTRE LES EXPERTS ET LES NON EXPERTS}

La corrélation observée entre les notes perceptives de la «beauté » et de la "santé » des écosystèmes semble tout à fait surprenante. En effet, il n'y a a priori pas de raisons objectives qui expliquent ce lien. Cette perception semble donc indiquer, chez les experts, un défaut d'objectivité dans leur appréciation de la « santé » des écosystèmes. Il se pourrait que ce résultat soit lié à une mauvaise compréhension du terme «santé » par la population des experts. Ce terme, emprunté au vocabulaire courant (c'est d'ailleurs la raison pour laquelle il avait été retenu), pourrait ne pas faire sens à une communauté de spécialistes en écologie : trop vague (Lackey, 2001 ; Rapport, 1995), difficile à appliquer compte tenu des propriétés inhérentes aux écosystèmes (Wicklum et Davies, 1995), voire connoté politiquement (Jamieson, 1995), ce terme a en effet fait l'objet de grandes controverses dans la littérature scientifique. On lui reproche avant tout d'être lié à la subjectivité de l'individu : il dépend en effet des valeurs que l'individu porte aux écosystèmes et à la nature. Si cela limite l'emploi de cette notion comme indicateur dans le cadre des politiques de gestion environnementale, elle est au contraire riche de sens dans le cadre d'une enquête de perception. Si l'on assume le fait que la notion de «santé » inclut une forte composante de subjectivité, elle s'avère défendable intellectuellement et valable sur le plan heuristique (Callicott, 1995).

Dans ce contexte, l'interprétation donnée au terme de "santé » par les experts ayant répondu à l'enquête pourrait indiquer davantage une vision « naturaliste » qu'une vision « fonctionnelle » des écosystèmes. En d'autres termes, ce n'est pas la normalité du fonctionnement de l'écosystème dans un contexte fonctionnel donné (contextes géologique, géomorphologique, chimique, lithologique, climatique, etc.) que les experts évaluent. Ils semblent davantage enclins à apprécier la santé des milieux au regard de leur aptitude à accueillir des espèces rares; rareté à laquelle est associée une certaine beauté. Cette perception «naturaliste » est très présente dans le champ professionnel de l'écologie, et en particulier en France (Bornette G., communication personnelle). Il est à ce titre tout à fait symptomatique que la majeure partie des écologues travaillent sur les tourbières et non sur les gravières, compte tenu de leur intérêt esthétique respectif.

Il semble donc primordial de mettre en place une campagne de communication auprès des experts écologues afin de rappeler les enjeux liés à la protection des milieux eutrophes dans certains contextes régionaux.

Vers une modélisation des préférences paysageres des écosystèmes aquatiques de bras morts

La mise en évidence de quatre types de plans d'eau représentatifs des perceptions sociales ouvre de nouvelles perspectives. En effet, à certaines caractéristiques physiques des écosystèmes aquatiques de bras morts, il est désormais possible d'associer des jugements esthétiques, voire des états émotionnels. Certaines variables ont ainsi été identifiées comme ayant un impact esthétique positif (transparence de l'eau, couleurs vives, contraste intense/hétérogénéité de la prise de vue, état dynamique du plan d'eau, algues aux contours bien définis), et d'autres, négatif (sédiments, opacité 
de l'eau, faible contraste/homogénéité de la prise de vue, couleurs sombres ou froides, algues aux contours peu définis, etc.). La constitution de ce panel de variables et la compréhension de leurs influences respectives sur la perception permet d'envisager une modélisation des perceptions paysagères des plans d'eau de bras morts (Cottet et al., 2009). Ainsi, à partir d'une photographie de plan d'eau de bras mort, et grâce aux résultats présentés ci-dessus, il est désormais possible de prévoir les réactions du public sur le plan esthétique. Les applications de ce travail semblent prometteuses : la modélisation des préférences paysagères, aboutissement de cette enquête, constitue un outil favorable à l'intégration d'objectifs sociaux dans les projets de restauration.

\section{Conclusion}

L'intégration des enjeux sociaux dans les projets de restauration demande une meilleure compréhension de la perception sociale des écosystèmes. En effet, entre les experts et le public doit s'établir un langage commun, base inéluctable à la mise en œuvre de projets concertés.

La typologie des plans d'eau de bras morts issue des perceptions sociales définie dans le cadre de cette enquête a ouvert la voie en ce sens. En effet, quatre types de plans d'eau de bras morts ont été caractérisés par le biais d'un " langage vernaculaire », décrivant à la fois les caractéristiques physiques du milieu (visuelles et écologiques) et les sentiments induits par la perception de ce type de milieu. La mise en relation des caractérisations objectives et subjectives d'un type d'écosystème aquatique donné permet d'anticiper les réactions du public face à un plan d'eau de bras mort. La mise en œuvre opérationnelle de cette prévision repose sur la construction d'un modèle de perception (Cottet et al., 2009) : à partir de variables explicatives mises en évidence dans le cadre de cette enquête, il semble possible de déduire les évaluations esthétiques concernant différents plans d'eau de bras morts. Véritable outil d'aide à la gestion, ce modèle de prévision des perceptions sociales pourrait être facilement utilisé pour une élaboration concertée des projets de restauration de bras morts.

\section{Bibliographie}

Amoros C., Bornette G., Henry C. P., 2000. A vegetation-based method for ecological diagnosis of riverine wetlands, Environmental Management, vol. 25, n 2, p. 211-227.

Arriaza M., Cañas-Ortega J. F., Cañas-Madueño J. A., Ruiz-Aviles P., 2004. Assessing the visual quality of rural landscapes, Landscape and Urban Planning, vol. 69, p. 115-125.

Barro S. C., Bright A. D., 1998. Public views on ecological restoration. A snapshot from the Chicago area, Restoration and Management, vol. Notes 16, p. 59-65.

Bazin P., Barnaud G., 2002. Du suivi à l'évaluation : à la recherche d'indicateurs opérationnels en écologie de la restauration, Revue d'écologie : la terre et la vie, vol. supplément 9, p. 201-224.

Berelson B. V., 1954. Content analysis, dans Lindzey G. (dir.), Handbook of social psychology. Adison wesley.

Blandin P., Bergandi D., 2000. A l'aube d'une nouvelle écologie?, La Recherche, vol. 332, p. 56-59.

Boulton A. J., 1999. An overview of river health assessment: philosophies, practice, problems and prognosis, Freshwater Biology, vol. 41, n² 2, p. 469-479.

Brody S. D., Highfield W., Peck M. B., 2005. Exploring the mosaic of perceptions for water quality across watersheds in San Antonio, Texas, Landscape and urban planning, vol. 73, p. 200-214.

Cairns J. J., 1995. Ecosocietal restoration. Reestablishing humanity's relationship with natural systems, Environment, vol. 37, $n^{\circ}$ 5, p. 4-9; 30-33.

Callicott J. B., 1995. A review of some problems with the concet of ecosystem health, Ecosystem Health, vol. $1, n^{\circ} 2$, p. 101-112.

Charles L., Kalaora B., 2003. L’ingénierie écologique entre écologie, technique et aménagement : des enjeux durables, dans LévÊQue C., VAn Der Leeuw S. (dir.), Quelles natures voulons-nous? Pour une approche socio-écologique du champ de l'environnement, Paris, Elsevier, p. 225-235. 
Cossin M., 2008. Caractérisation paysagère de quelques cours d'eau de taille moyenne des bassins du Rhône et de la Loire: Éléments méthodologiques pour une gestion durable des corridors fluviaux, thèse de doctorat. Géographie et aménagement, Université Jean-Moulin Lyon 3, 298 p.

Cottet M., Piégay H., Honegger A., 2009. Modélisation des préférences esthétiques : vers la prise en compte des perceptions dans les projets de restauration écologique de bras morts dans FoLTÊTE J.-C. (dir.), Les neuvième rencontres de Théoquant, France, Besançon, 4-6 mars 2009 [http://thema.univ-fcomte.fr/theoq/ pdf/2009/TQ2009\%20ARTICLE\%2026.pdf].

DANiEL T. C., 2001. Whither scenic beauty? Visual landscape quality assessment in the 21 st century, Landscape and urban planning, vol. 54, p. 267-281.

Daniel T. C., Boster R. S., 1976. Measuring Landscape Aesthetics: The Scenic Beauty Method, USDA Forest Service Research, Rocky Mountian Forest and Range Experiment Station. Paper RM-167, 66 p.

De Vanssay B., 2003. Les représentations de l'eau, Vertigo, vol. 4, n 3, Hors-série $1^{\text {er }}$ décembre 2003, [En ligne] [http://vertigo.revues.org].

Dearden P., 1985. Philosophy, theory and method in landscape evaluation, Canadian Geographic, vol. 29, p. 263-265.

Donadieu P., 2002. Les références en écologie de la restauration, Revue d'écologie : la terre et la vie, vol. supplément 9, p. 109-120.

Giordano R., Passarella G., Uricchio V. F., Vurro M., 2005. Fuzzy cognitive maps for issue identification in a water resources conflict resolution system, Physics and Chemistry of the Eearth, vol. 30, p. 463-469.

Gobster P. H., Barro S. C., 2000. Negociating nature. Making restoration happen in an urban park context, dans Gobster P. H., Hull R. B. (dir.), Restoring nature. Perspectives from the social sciences and humanities, Washington D.C., Island press, p. 185-207.

Gobster P. H., Nassauer J. I., Daniel T. C., Fry G., 2007. The shared landscape: what does aesthetics have to do with ecology?, Landscape Ecology, vol. 22, p. 959-972.

Herzog T. R., 1985. A cognitive analysis of preference for waterscapes, Journal of Environmental Psychology, vol. 5, p. 225-241.

Herzog T. R., Bosley P. J., 1992. Tranquility and preference as effective qualities of natural environments, Journal of Environmental Psychology, vol. 12, p. 115-127.

Higgs E. S., 1997. What is good ecological restoration?, Conservation Biology, vol. 1 1, n² 2, p. 338-348.

House M. A., SAngster E. K., 1991. Public perceptions of river corridor management, Journal of IWEM, vol. 5, p. 312-317.

Ibery B. W., Foster I. D. L., Donogue P. J., 1982. Perception and water quality: a geographical perspective, Progress in Physical Geography, vol. 6, n 4, p. 524-540.

Jamieson D., 1995. Ecosystem health: some preventative medicine, Environmental values, vol. 4, p. 333-344.

Junker B., Buchecker M., 2008. Aesthetic preferences versus ecological objectives in river restorations, Landscape and Urban Planning, vol. 85, p. 141-154.

Кон J., 1988. An ecological aesthetic, Landscape Journal, vol. 7, p. 177-191.

LACKey R. T., 2001. Values, policy, and ecosystem health, BioScience, vol. 51, nº 6, p. 437-443.

Le Floc'h E., Aronson J., 1995. Écologie de la restauration. Définition de quelques concepts de base, Natures-Sciences-Sociétés, vol. vol 3, nnuméro spécial, p. 29-35.

LE LAY Y., 2007. Les hommes et le bois en rivière. Représentations, pratiques et stratégies de gestion dans le cadre de l'entretien des cours d'eau, Thèse de doctorat. Géographie et aménagement, Université Jean-Moulin Lyon 3, 570 p.

Le Lay Y., Piégay H., 2007. Le bois mort dans les paysages fluviaux français : éléments pour une gestion renouvelée, Espace géographique, vol. 36, n 1, p. 51-64.

Le Lay Y., Piégay H., Cossin M., 2005. Les enquêtes de perception paysagère à l'aide de photographies, choix méthodologiques et exemples en milieu fluvial, dans Foltête J.-C. (dir.), Septièmes rencontres de Théo Quant, Besançon.

Le Lay Y., Piégay H., Rivière-Honegger A., (en préparation). La rivière en tresses : un paysage atypique et mésestimé. 
Marylise Cottet, Anne Rivière-Honnegger, Hervé Piegay

Linton D., 1968. The assessment of scenery as a natural resource, Scottish Geographical Magazine, vol. 84, p. 219-238.

Lowenthal D., Prince H. C., 1965. English landscape tastes, Geographical Review, vol. 55, n² 2, p. 186-222.

Lukasiewicz E., Fischler C., Setbon M., Flahault A., 2001. Comparaison de trois échelles d'évaluation de la perception des risques sanitaires, Revue d'épidémiologie et de santé publique, vol. 49, p. 377-385.

Millennium Ecosystem Assessment, 2005. Ecosystems and human well-being: wetlands and water, Institute W. R., Washington D.C., 68 p.

Moser G., 1984. Water quality perception, a dynamic evaluation, Journal of Environmental Psychology, vol. 4, p. 201-210.

Mouratiadou I., Moran D., 2007. Mapping public participation in the Water Framework Directive: a case study of the Pinios River Basin, Greece, Ecological Economics, vol. 62, p. 66-76.

Mutz M., Piégay H., Gregory K. J., Borchardt D., Reich M., Klaus S. K., 2006. Perception and evaluation of dead wood in streams and rivers by German students, Limnologica, vol. 36, p. 110-118.

Nassauer J. I., 1992. The appearance of ecological systems as a matter of policy, Landscape Ecology, vol. 6, $n^{\circ} 4$, p. 239-250.

Nassauer J. I., 2004. Monitoring the success of metropolitan wetland restorations: cultural sustainability and ecological function, Wetlands, vol. 24, p. 756-765.

Nassauer J. I., Kosek S. E., Corry R. C., 2001. Meeting public expectations with ecological innovation in riparian landscapes, Journal of the American Water Resources Association, vol. 37, n 6, p. 1439-1443.

PARsons R., 1995. Conflict between ecological sustainability and environmental aesthetics: Conundrum, canärd or curiosity, Landscape and Urban Planning, vol. 32, p. 227-244.

Piégay H., Gregory J., Bondarev V., Chin A., Dahlstrom N., Elosegi A., Gregory S., Joshi V., Mutz M., Rinaldi M., Wyzga W., Zawiejska J., 2005. Public perception as a barrier to introducing wood in rivers for restoration purposes, Environmental Management, vol. 36, n 5, p. 665-674.

Raffetto J., 1993. Perceptions of ecological restoration in urban parks, dans Gobster P. H. (ed.), Managing Urban and High-Use Recreation Settings, General Technical Report NC-163, United States Department of Agriculture, p. 61-67.

RApport D. J., 1995. Ecosystem health: exploring the territory, Ecosystem Health, vol. 1, n 2, p. 5-13.

Real E., Arce C., Sabucedo J. M., 2000. Classification of landscapes using quantitative and categorical data, and prediction of their scenic beauty in North-Western Spain, Journal of Environmental Psychology, vol. 20, p. 355-373.

Shafer E. L., 1969. Perception of natural environments, Environment and Behavior, vol. 8, p. 71-82.

Shuttleworth S., 1980. The use of photographs as an environmental presntation medium in landscape studies, Journal of Environmental Management, vol. 11, p. 61-76.

Simberloff D., 1998. Flagships, umbrellas and keystones: is single-species management passé in the landscape era?, Biological Conservation, vol. 83, p. 247-257.

Smith D. G., Davies-Colley R. J., 1992. Offsite measurement of the visual clarity of waters, Water Resources Bulletin, vol. 28, p. 951-957.

SpIRn A. W., 1988. The poetics of city and nature: towards a new aesthetic for urban design, Landscape Journal, vol. 7, p. 108-126.

STAmps A. E., 1992. Bootstrap investigation of respondant sample size for environmental preference, Perceptual and motor skills, vol. 75 , p. 220-222.

STEINITZ C., 1990. Toward a sustainable landscape with high visual preference and high ecological integrity: the loop road in Acadia National Park, U.S.A., Landscape and urban planning, vol. 19, p. 213-250.

Steinwender A., Gundacker C., Wittmann K. J., 2008. Objective versus subjective assessements of environmental quality of standing and running waters in a large city, Landscape and urban planning, vol. 84, p. 116-126.

Suter G. W., 1993. A critique of ecosystem health concepts and indexes, Environmental Toxicology and Chemistry, vol. 12, p. 61-79.

Thorne J. et Huang C.-S., 1991. Toward a landscape ecological aesthetic: methodologies for designers and planners, Landscape and Urban Planning, vol. 21, p. 61-79. 
Trémolières M., Schnitzler A., Carbiener D., 2002. Quel système de référence pour la restauration des systèmes alluviaux rhénans?, Revue d'écologie : la terre et la vie, vol. supplément 9, p. 131-145.

Ulrich R. S., 1981. Natural versus urban scenes. Some psychophysiological effects, Environment and Behavior, vol. 13, p. 523-556.

Vining J., Orland B., 1989. The video advantage: a comparison of two environmental representation techniques, Journal of Environmental Management, vol. 29, p. 275-283.

Wicklum D., Davies R. W., 1995. Ecosystem health and integrity?, Canadian Journal of Botany, vol. 73, p. 997-1000.

Yamashita S., 2002. Perception and evaluation of water in landscape: use of photo-projective method to compare child and adult residents' perceptions of a Japanese river environment, Landscape and Urban Planning, vol. 62, p. 3-17.

YANG B., Brown T. C., 1992. A cross-cultural comparison of preferences for landscape styles and landscape elements, Environment and Behavior, vol. 24, p. 471-507.

Zube E., Simcox D. E., Law C. S., 1987. Perceptual landscape simulations: history and prospect, Landscape Journal, vol. 6, p. 62-80.

Cet article a été reçu le 28 avril 2009 et définitivement accepté le 15 juin 2010 
planche XI (Émilie BOURGET, Laurence LE DÛ-BLAYO - Définition d'unités paysagères par télédétection...)

(Marylise COTTET et al. - Mieux comprendre la perception des paysages de bras morts...)

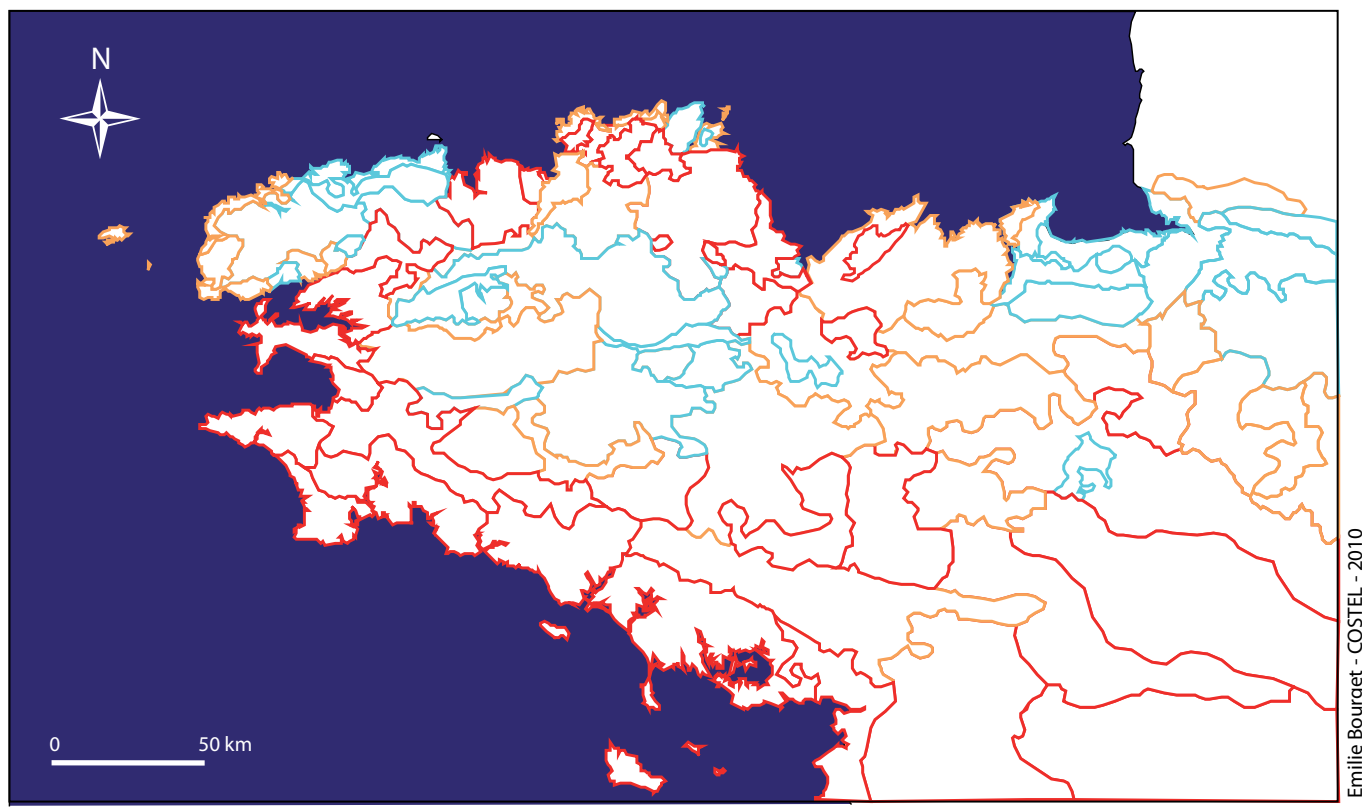

Figure 11 : Typologie de l'incertitude des limites des unités de paysage de synthèse units boundaries

Typology of uncertainty of synthesis landscape

incertitude faible

__ incertitude moyenne incertitude forte
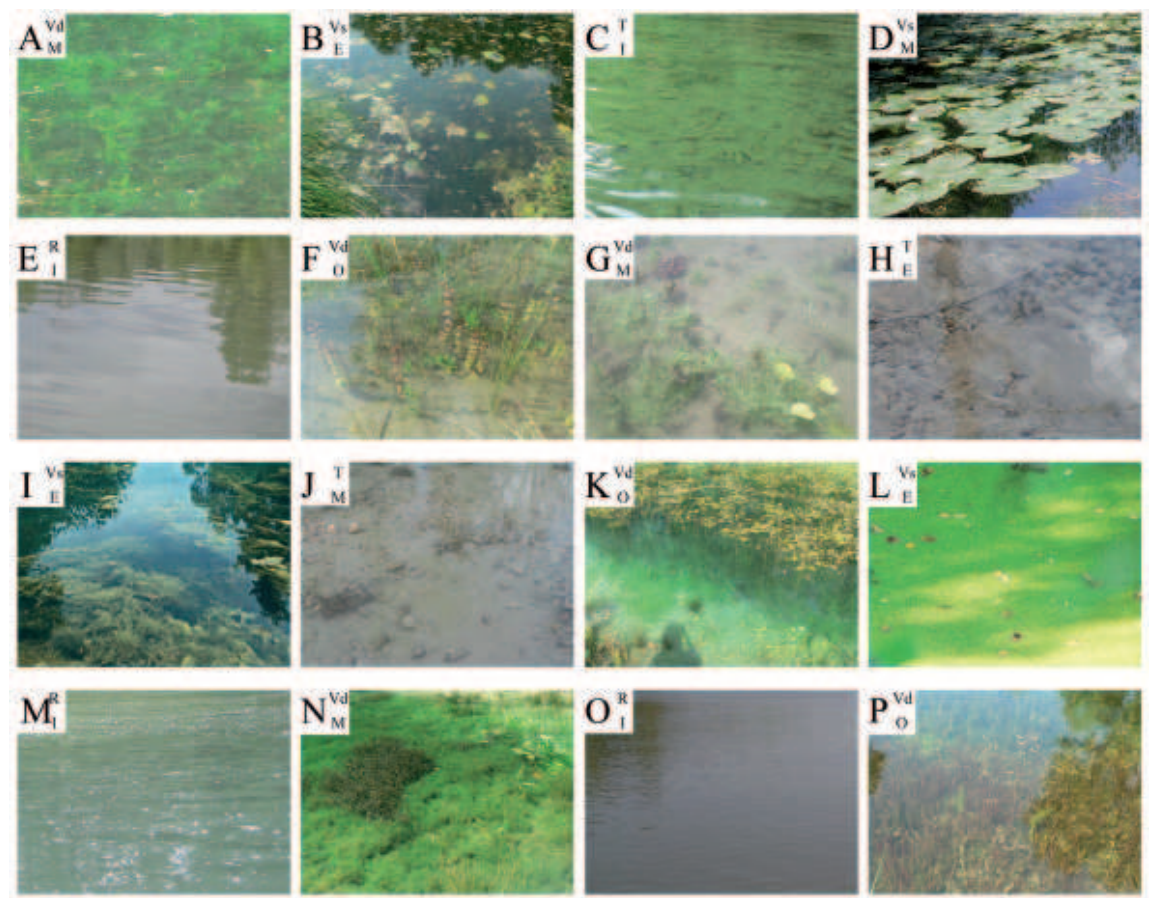

Photo 1 : Photographies des plans d'eau de bras morts utilisées dans le photo-questionnaire Photographs of the floodplain lakes waters used in the photo-questionnaire 


\begin{tabular}{|c|c|c|c|c|}
\hline Classe & Plans d'eau & Thèmes $\mathrm{q}$ & lificatifs & Beauté \\
\hline 1 & & 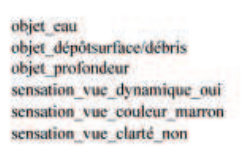 & 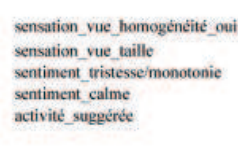 & $4,1 / 10$ \\
\hline 2 & & 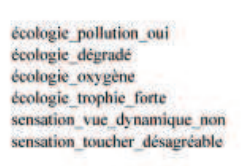 & $\begin{array}{l}\text { sensation odcur désagertable } \\
\text { sentiment beauté non } \\
\text { sentiment dangeref inquietude } \\
\text { sentiment degegoit } \\
\text { sentiment proprete non }\end{array}$ & $3,7 / 10$ \\
\hline 3 & & 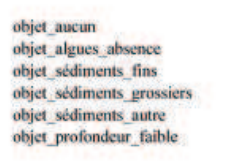 & 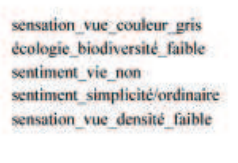 & $4,5 / 10$ \\
\hline 4 & & 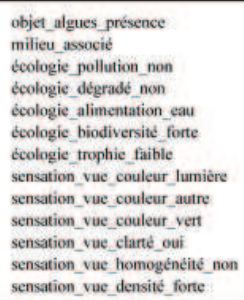 & 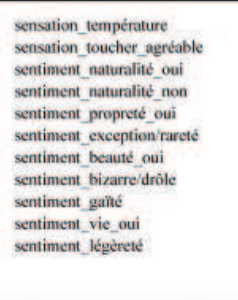 & $7,1 / 10$ \\
\hline
\end{tabular}

Figure 6 : Description des classes issues de la CAH et moyenne d'esthétique correspondante HAC's classes description and corresponding aesthetic means 